\title{
A model of neuropathic pain in brachial plexus avulsion injury and associated spinal glial cell activation
}

\section{Ao-Lin Hou \\ Wen-Dong Xu}

Department of Hand Surgery, Huashan Hospital, Fudan University, Shanghai 200040, China
Correspondence: Wen-Dong Xu Department of Hand Surgery, Huashan Hospital, No. 12 Wulumuqi Road, Shanghai 200040, China Tel +86 2I 52888732

Fax +86 2I 52888735

Email xuwendong_huashan@163.com
This article was published in the following Dove Press journal: Journal of Pain Research

Background: Neuropathic pain is a common and intractable sequel of brachial plexus injury. Materials and methods : To investigate the underlying mechanisms, we established a unique model of neuropathic pain in rats by creating brachial plexus avulsion injury.

Results: Behavioral test of mechanical stimulation suggested that all rats developed neuropathic pain, and the pain thresholds of bilateral hind limbs significantly decreased. GFAP expression in the cervical spinal cord appeared on day 1 post-injury and increased on day 4 . Ionized calcium-binding adaptor molecule 1 expression appeared on day 1 post-injury and increased until day 28. Therefore, the brachial plexus avulsion injury model can imitate the development of neuropathic pain and maintain it.

Conclusion: The activation of astrocyte and microglia in the spinal cord plays a key role in the mechanism and treatment of neuropathic pain.

Keywords: neuropathic pain, new model, astrocyte, microglia

\section{Introduction}

Brachial plexus avulsion injury (BPAI) is a most devastating peripheral nerve injury in the upper extremity. When upper extremity suddenly suffer powerful force, especially in traffic accident, all nerve roots from $\mathrm{C} 5$ to $\mathrm{T} 1$ are completely avulsed from the cervical spinal cord. In this injury, the rootlets of the C5-T1 spinal nerves connecting the central nervous system (CNS) with the peripheral nerves are divided, which makes the whole upper limb paralyzed. ${ }^{1}$ Obviously, it is impossible to repair by direct repair. These patients are suffering from negative effects of BPAI on sensorimotor functions and quality of life.

In addition to sensorimotor dysfunctions, a number of BPAI patients suffer from neuropathic pain. It is a serious complication, which is long lasting, hard to relieve, and drug resistant. It was reported that patients with BPAI were easier to sustain neuropathic pain than those with other peripheral nerve injuries. ${ }^{2}$ According to the literature, almost $67 \%$ of BPAI patients would develop neuropathic pain. ${ }^{3}$ Previous studies indicated that neuropathic pain following BPAI is difficult to treat in clinical practice, and most treatments could not relieve the pain effectively. ${ }^{4}$ Therefore, it is essential to explore its underlying mechanisms, which is not fully understood so far.

Mounting studies have demonstrated the role of spinal glial cell in the occurrence and development of neuropathic pain. ${ }^{5}$ Garrison et $\mathrm{al}^{6}$ indicated for the first time that the activation of spinal glial cells and astrocyte is associated with hyperalgesia. Paszcuk et $\mathrm{al}^{7}$ also reported that glial cells played an important role in modulating BPAI-induced 
neuropathic pain. Thus, we speculated that glial cells may largely involve in the process of neuropathic pain after BPAI.

In the present study, we established a modified BPAI model in adult rats with neuropathic pain confirmed by behavioral tests. The immunohistochemistry studies of glial fibrillary acidic protein (GFAP; for astrocytes) and ionized calcium-binding adaptor molecule 1 (Iba-1; for microglia) were performed at different post-injury intervals to investigate the change in glial cells in the spinal cord during the process of neuropathic pain formation. This study aimed to establish a modified BPAI model and demonstrate this model can induce neuropathic pain assessing by the activation of spinal glial cell.

\section{Materials and methods Animals}

A total of 60 female adult Sprague Dawley (SD) rats (weight, 180-200 g; age, 6 weeks) were divided into seven groups: one control group $(n=12)$ and six BPAI groups ( $n=8$ per group). Rats were grouped into six BPAI groups by different time points for immunohistochemistry studies as day 1, 4, 7, 14, 21 and 28, respectively. Unlike previous models that are often performed by the anterior cervical approach, ${ }^{7}$ we designed a modified posterior cervical procedure to produce BPAI.

The procedures were approved by the Animal Research Committee, Fudan University. All experiments were performed in accordance with the guide for the Care and Use of Laboratory Animals as described by the US National Institutes of Health, and all the experiments were designed to minimize both the number of animals used and their suffering.

\section{Surgical procedure of BPAI}

The BPAI model was established under anesthesia induced by a $10 \%$ chloral hydrate solution injection intraperitoneally. The rat was placed in the prone position. Under sterile conditions, a posterior midline incision was made on the back of the neck. The spinous process of the second thoracic vertebra was found, and the left side muscle was parted to expose the facet of 4 - 11 vertebra. Hemilaminectomy of left C4-T1 was performed by micro-dissecting scissors, and the dorsal roots of $\mathrm{C} 5-\mathrm{T} 1$ were exposed. Then, the dorsal roots of C5-T1 were avulsed from the spinal cord, and the ventral roots were exposed. The ventral roots were also avulsed by the same approach. The surgery was performed under the operating microscope. The key steps were shown in Figure 1.

Sham operation was performed in the control group. Hemilaminectomy of C4-T1 and exposure of brachial plexus nerves (C5, C6, C7, C8, and T1) on the left side were performed in the control group, consistent with that in the experimental group. Then, the incision was closed without brachial plexus avulsion.

\section{Behavioral tests}

The behavioral tests were performed in accordance with previous studies. ${ }^{8-11}$ Before the test, rats were habituated in the testing environment for 30 minutes. All the behavioral tests were carried out blindly. All rats in six BPAI groups were tested at each post-injury time point.

\section{Mechanical pain tests}

Mechanical pain was assessed by the method described previously by using Von Frey Filament. ${ }^{8,11}$ Rats were placed on a metal mesh floor $\left(0.8 \times 0.8 \mathrm{~cm}^{2}\right.$ cells $)$ covered by a transparent plastic box that was kept at $30 \mathrm{~cm}$ above the floor. After approximately 15 minutes of adaption, we fixed its hind limb with a nipper when the hind limb fell into the grid. The Von Frey filament was used to apply a linearly increasing pressure on the hind limb until the rat withdrew it. The mechanical pain threshold were recorded on days 0 (pre-injury), 1, 4, $7,1,21$, and 28 post-injury. The stimulating duration was 4 seconds, and the interval between stimulations was $30 \mathrm{sec}-$ onds. The mechanical pain threshold described as the lowest force that evoked a consistent brisk withdrawal response. The $50 \%$ mechanical pain threshold was evaluated and compared with the pre-injury data. The data on day 0 (pre-injury) were set as baseline.

\section{Thermal pain tests}

Thermal pain threshold was assessed with thermal pain stimulation system as previously described. ${ }^{8-10}$ The rat was placed in a glass box with a bottom. The radiant heat source was positioned under the glass floor directly beneath the hind limb. While the beam intensity was set at 5 , the time when the rat withdrew the paw was recorded. The thermal pain threshold were also recorded on days 0 (pre-injury), 1, 4, 7, 1, 21 , and 28 post-injury. Thermal pain threshold was the duration when rats showed a withdrawal reaction. To avoid heat injury, the machine would be cut off after 25 seconds. Each rat was evaluated five times, and the interval was 3 minutes. The last three thermal pain thresholds were calculated and averaged. The data on day 0 (pre-injury) were set as baseline.

\section{Immunohistochemistry of GFAP (astrocyte) and lba-I (microglia)}

After behavioral tests at each interval, rats in each BPAI group and two rats in the control group were injected intraperitoneally 

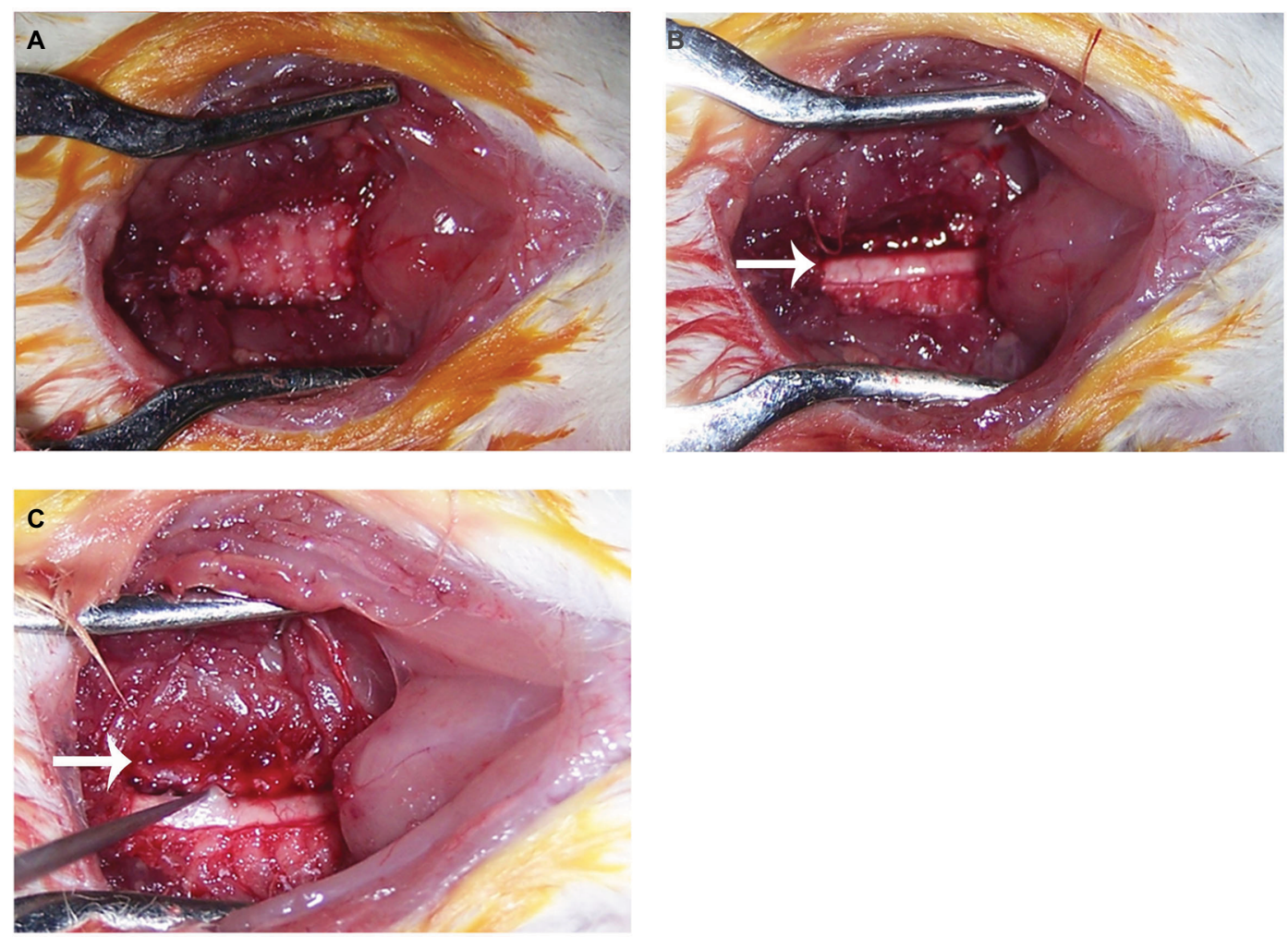

Figure I The surgical procedure of total brachial plexus avulsion injury via posterior cervical-thoracic approach in a rat. Notes: (A) The vertebral plate. (B) “ $\rightarrow$ "shows the left half of the spinal cord. (C) “ $\rightarrow$ "shows avulsed left nerve roots.

with excessive $10 \%$ chloral hydrate solution and then transcardially perfused with $250 \mathrm{~mL}$ of $0.9 \%$ saline, followed by $4 \%$ paraformaldehyde in phosphate buffer $(0.1 \mathrm{M}, \mathrm{pH} 7.4)$ for 2 hours. Cervical enlargement was resected, post-fixed in $4 \%$ paraformaldehyde for 4 hours, and dehydrated using graded sucrose. The tissue samples were frozen in tissue-freezing medium and sliced into $40 \mu \mathrm{m}$ transverse sections on a cryostat. Every sixth section was collected and placed in the protective fluid. Approximately 70 sections of the sample were harvested from the rat. All sections were rinsed three times for 10 minutes each in 0.01M PBS (pH 7.4) and then incubated with blocking buffer (10\% goat serum [KPL, 71-00-27] and 0.3\% Triton $\mathrm{X}-100$ in $0.01 \mathrm{M}$ PBS) overnight for $4^{\circ} \mathrm{C}$. Primary antibody, rabbit anti-GFAP (1:1,000; Chemicon Company, Temecula, CA, USA), and rabbit anti-Iba-1 (1:1,000; Chemicon Company) were added to the blocking buffer and incubated with sections at $4^{\circ} \mathrm{C}$ for 48 hours. The blocking buffer was replaced, and the secondary antibody (fluorescein isothiocyanate [FITC]-conjugated goat-anti-rabbit IgG, 1:2,000; Wuhan Boster Biological Technology, Wuhan, China; BA1105) was added and incubated with sections at $37^{\circ} \mathrm{C}$ for 2 hours. Activated glial cells were observed with a Leica fluorescence microscope. Five random areas in the spinal dorsal horn of each sample were examined at $\times 200$ magnification. The average number of the activated astrocyte and microglia in these five areas was calculated.

\section{Data analyses}

SPSS 15.0 was used for the statistical analyses. These data were presented as mean \pm SEM. Statistical significance between groups was determined by using one-way ANOVA followed by Student-Newman-Keuls (SNK) test. In the same group, paired sample $t$-test was used to test the significant difference. $P<0.05$ was considered as statistically significant.

\section{Results \\ Behavioral results}

\section{The basic behavioral observation}

The left forelimb of all rats in the BPAI groups was totally paralyzed. The feeding behavior was not influenced. No evidence of cervical instability was observed after C5-T1 hemilaminectomy. After BPAI, three rats showed self-mutilation behavior of biting the paralyzed limb. The self-damaged area ranged from nails to the elbow. No rat showed severe impairment. 
Mechanical stimulation-induced pain of bilateral hind limbs

Mechanical pain thresholds of bilateral hind limbs were $13 \pm 3.16 \mathrm{~g}$ (left) and $14.3 \pm 6.21 \mathrm{~g}$ (right) at baseline in all rats. At day 1 after the BPAI, the mechanical pain thresholds of bilateral hind limbs significantly decreased to $5.67 \pm 0.82 \mathrm{~g}$ (left) and $5.67 \pm 0.82 \mathrm{~g}$ (right) in all the six groups comparing with baseline data $(P<0.05)$. The threshold in 10 individuals even decreased to $2-4 \mathrm{~g}$. The mechanical pain threshold in all rats increased to $6 \pm 1.26 \mathrm{~g}$ (left) and $7 \pm 1.67 \mathrm{~g}$ (right) after 6 days. On day 28, the mechanical pain threshold of bilateral hind limbs was $7 \pm 1.10 \mathrm{~g}$ (left) and $7 \pm 1.10 \mathrm{~g}$ (right), which was significantly lower than the baseline value $(P<0.05)$. The threshold in the control group was unchanged during the 28 days. The mechanical pain thresholds of bilateral hind limbs during the 28 days are shown in Figure 2A.

\section{Thermal stimulation-induced pain of bilateral hind} limbs

BEAM value was set at 5 , and the withdrawal time was 9.75 \pm 1.37 seconds (left) and $9.71 \pm 3.11$ seconds (right) at baseline in all rats. Compared with the change of mechanical pain threshold, thermal pain threshold slightly decreased to $8.85 \pm 1.3$ seconds (left) and $8.39 \pm 1.80$ seconds (right) at day 1 after BPAI and recovered to $8.84 \pm 1.68$ seconds (left) and $9.64 \pm 2.38$ seconds (right) at 2 weeks after the procedure. The threshold of control group was unchanged during the 28 days. No statistical significance was found when comparing with the baseline data $(P>0.05)$. The thermal thresholds of bilateral hind limbs at each time point are shown in Figure 2B.

\section{Immunohistochemistry study of GFAP}

The expression of GFAP was elevated in the spinal cord of the paralyzed side on post-BPAI on day 1 (mean, $59 / \mathrm{mm}^{2}$ ) and increased on day 4 (mean, $74 / \mathrm{mm}^{2}$ ). The astrocytes were

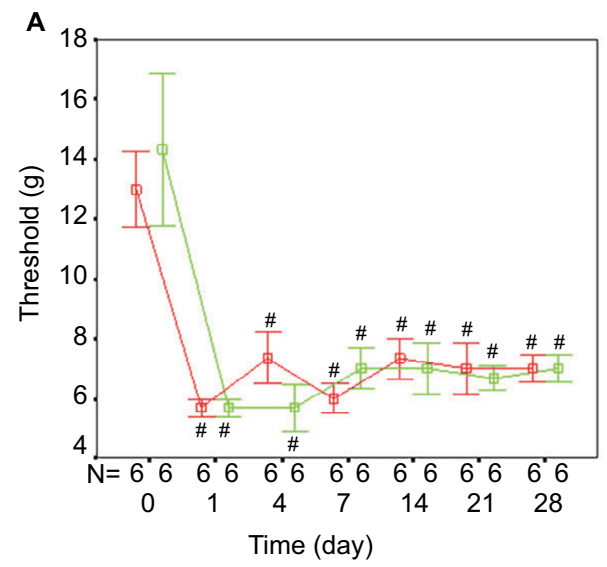

still activated and expressed as GFAP until post-operative day 28 (mean, 56/ $\mathrm{mm}^{2}$ ). The expression of GFAP significantly increased on the paralyzed side, either comparing with the control group (mean, $25 / \mathrm{mm}^{2} ; P<0.05$ ) or the intact side (mean, $23 / \mathrm{mm}^{2} ; P<0.05$ ). The GFAP expressions at each time point are shown in Figure 3.

\section{Immunohistochemistry study of lba- I}

In BPAI rats, the expression of Iba-1 in the bilateral spinal cord on day 1 post-BPAI was $50 / \mathrm{mm}^{2}$ (averaged; left) and 41 / $\mathrm{mm}^{2}$ (right). Compared with the control group $\left(25 / \mathrm{mm}^{2}\right.$ on the left side and $26 / \mathrm{mm}^{2}$ on the right side), BPAI-induced Iba-1 expression significantly increased and continuously increased on both sides on day $4\left(50 / \mathrm{mm}^{2}, 32 / \mathrm{mm}^{2}\right), 7\left(41 / \mathrm{mm}^{2}, 37 / \mathrm{mm}^{2}\right)$, $14\left(47 / \mathrm{mm}^{2}, 41 / \mathrm{mm}^{2}\right), 21\left(41 / \mathrm{mm}^{2}, 35 / \mathrm{mm}^{2}\right)$, and $28\left(40 / \mathrm{mm}^{2}\right.$, $28 / \mathrm{mm}^{2}$ ). The expression of Iba-1 significantly increased on both sides in the BPAI rats when compared with the control group $(P<0.05)$. On day 1,4 , and 28 , when compared with the intact side $\left(41 / \mathrm{mm}^{2}, 32 / \mathrm{mm}^{2}\right.$ and $28 / \mathrm{mm}^{2}$, respectively), the expression of Iba-1 significantly increased on the paralyzed side $\left(50 / \mathrm{mm}^{2}, 50 / \mathrm{mm}^{2}\right.$ and $40 / \mathrm{mm}^{2}$, respectively; $\left.P<0.05\right)$. The Iba- 1 expression at each time point is shown in Figure 4.

\section{Discussion and conclusion}

This study introduced a modified model of neuropathic pain by BPAI in adult rats. Immunohistochemistry study on GFAP (astrocyte) and Iba-1 (microglia) of the spinal cord sections and behavioral observations were performed. The outcomes demonstrated that our rat model can simulate BPAI and may be used to investigate the mechanism of neuropathic pain.

\section{Discussion}

\section{The modified rat model}

The classified neuropathic pain model was proposed by Gary and Bennett in $1998 .{ }^{12}$ They reported that chronic constriction
B

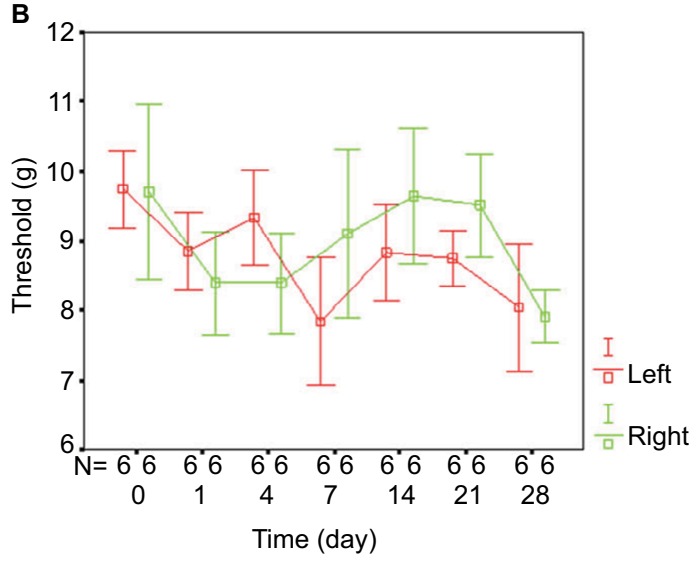

Figure 2 The mechanical pain and thermal pain thresholds after BPAI.

Notes: (A) Mechanical pain threshold values at each time point following BPAI. (B) Thermal pain threshold values at each time point following BPAI $\left({ }^{P}<<0.05\right)$.

Abbreviation: BPAl, brachial plexus avulsion injury. 

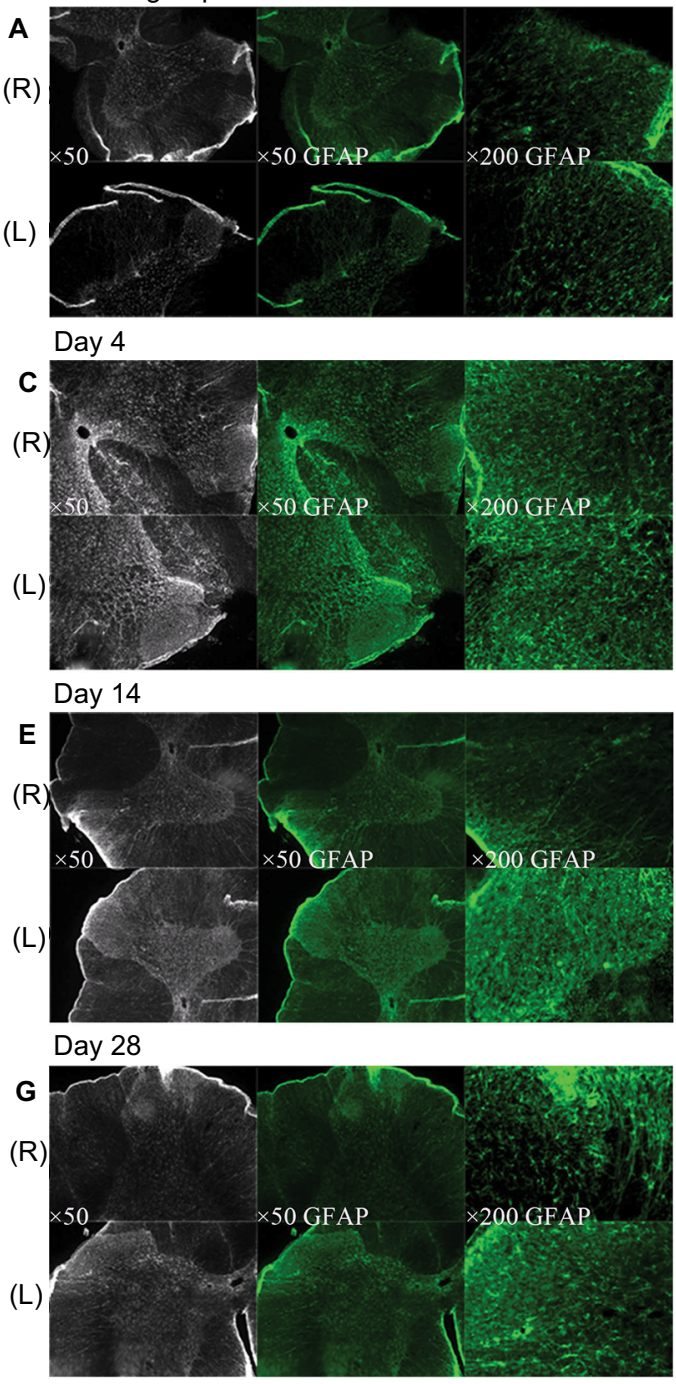

\section{Day 1}

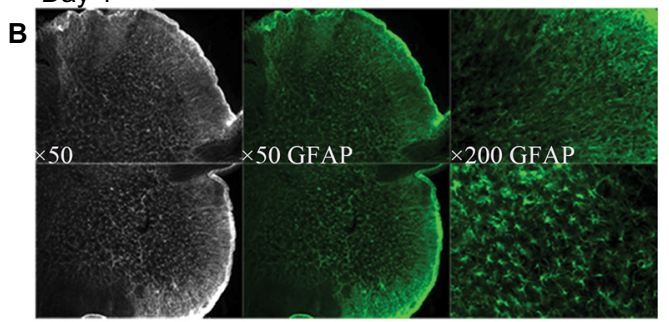

Day 7

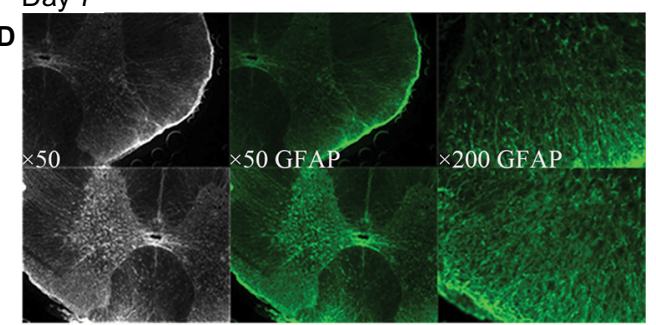

Day 21
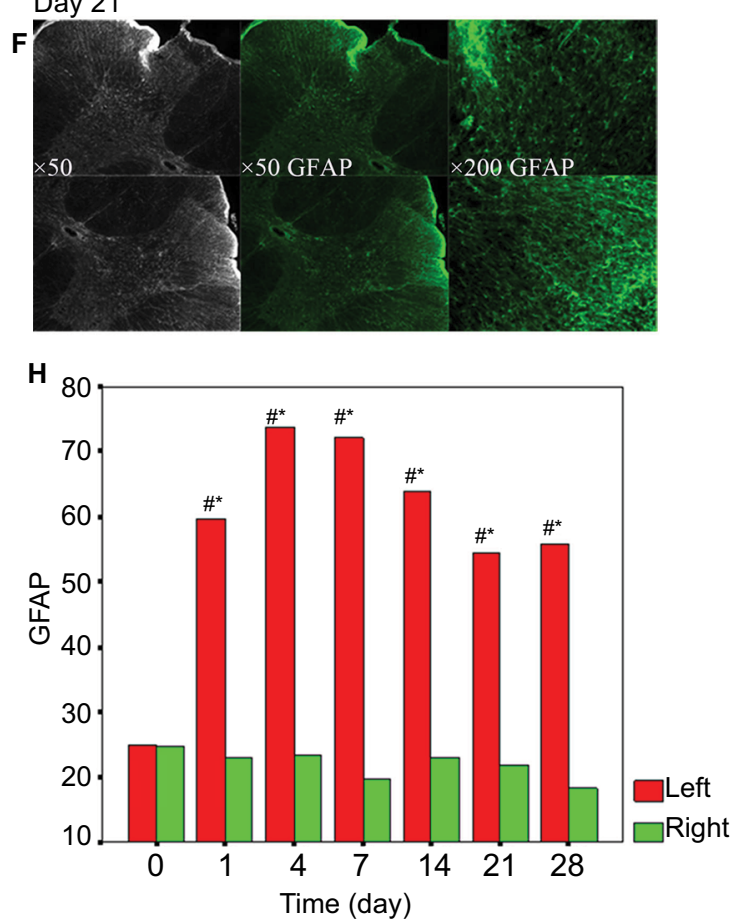

Figure 3 Immunohistochemistry for GFAP in bilateral spinal dorsal horns in normal rats and BPAI rats.

Notes: (A) GFAP expression of the normal group. (B-G) GFAP expression of the experimental group at I, 4, 7, I4, 2 I, and 28 days after BPAl. (H) Comparison of changes in the GFAP expression at $0,1,4,7,14,21$, and 28 days between the two groups. \#Significant differences between the two groups, $P<0.05$; *significant differences between the two sides in the same group, $P<0.05$.

Abbreviations: BPAl, brachial plexus avulsion injury; GFAP, glial fibrillary acidic protein; L, left side, R, right side.

injury (CCI) rat model, which showed symptoms similar to that of human neuropathic pain, mainly manifested spontaneous pain and hyperalgesia on thermal and mechanical stimulation. Except for the CCI, partial sciatic nerve ligation (PSNL), spinal nerve ligation (SNL), spared nerve injury (SNI), and rhizotomy models have also been used for experimental investigations on neuropathic pain. ${ }^{9,13-15}$ However, these models simply imitated the symptoms of neuropathic pain of human, but not the underlying pathogenesis.
Based on our clinical experience, we found that a great number of BPAI patients were suffering from neuropathic pain. Therefore, we established BPAI model in adult rats to verify its feasibility in imitating the symptoms and course of neuropathic pain. Previous researches tended to use the anterior cervical-thoracic approach to establish BPAI model. In clinical practice, branchial plexus roots are avulsed in the vertebral canal. However, it is difficult to determine whether the avulsion site of nerve root is pre- or post-ganglionic. 


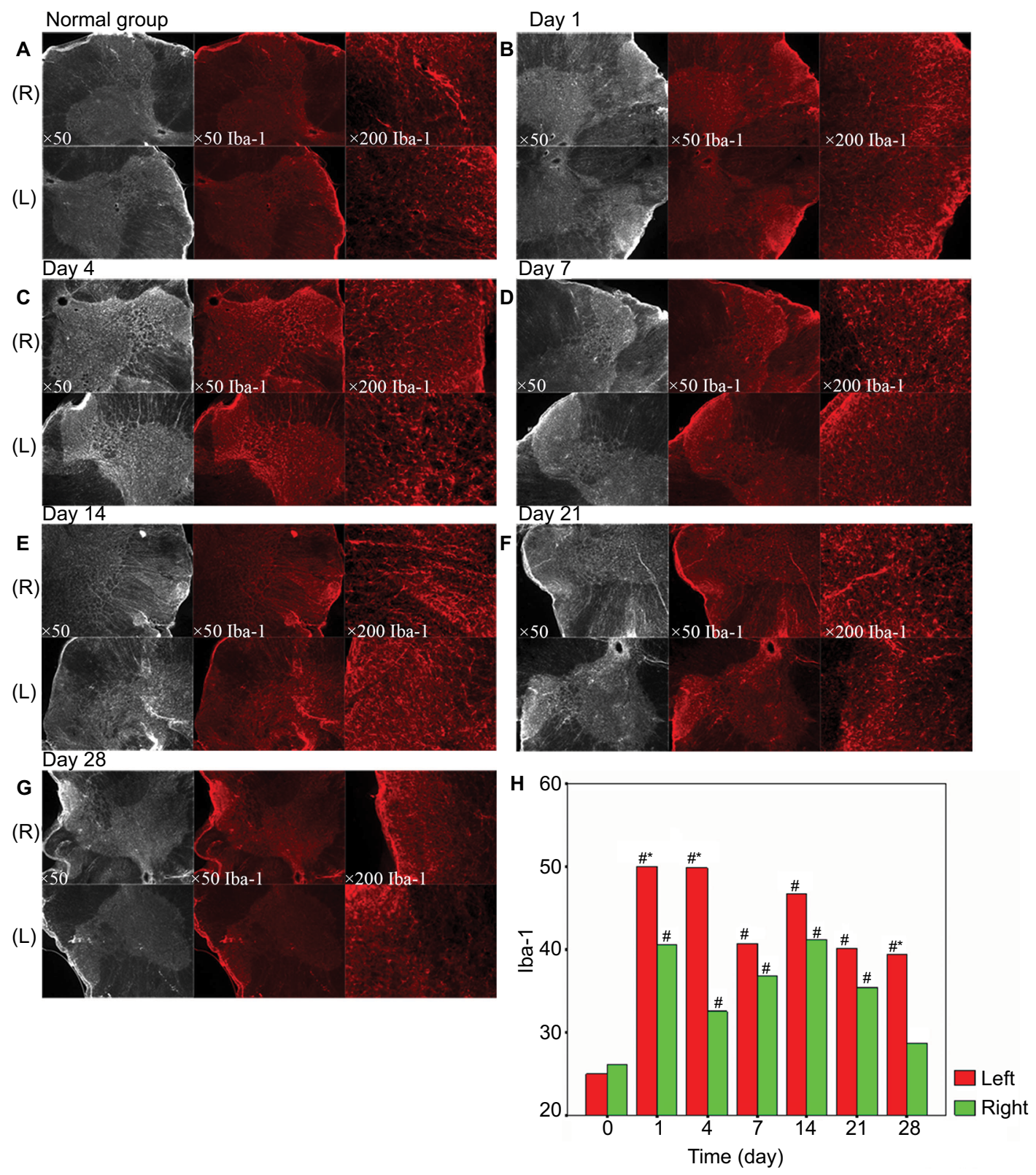

Figure 4 Immunohistochemistry for lba-I in bilateral spinal dorsal horn in normal rat and BPAI rats.

Notes: (A) Iba-I expression of the normal group. (B-G) lba-I expression of the experimental group at I, 4, 7, I4, 2 I, and 28 days after BPAl. (H) Comparison of changes in the lba-I expression at $0,1,4,7,14,21$, and 28 days between the two groups. "Significant differences between the two groups, $P<0.05$; *significant differences between the two sides in the same group, $P<0.05$.

Abbreviations: BPAI, brachial plexus avulsion injury; Iba-I, ionized calcium-binding adaptor molecule I; L, left side, R, right side.

Except that, the lower brachial plexus nerves were difficult to expose, and the subclavian vein might be injured. ${ }^{7}$ In our study, we used posterior cervical-thoracic approach to establish the rat model. First, we could avulse the nerve root under direct vision at pre-ganglionic lesion. Second, the success rate and accuracy rate of the posterior cervical-thoracic approach is higher. In our experiment, we observed that all rats in the BPAI groups showed complete paralysis in the left forelimbs. No significant instability of the cervical vertebra was observed after the hemilaminectomy of C5-T1.

\section{BPAl-induced neuropathic pain}

In previous studies, the indexes for assessing neuropathic pain in animals were spontaneous pain and allodynia. ${ }^{13}$ The behavior of spontaneous pain in the paralyzed limb was hard to assess quantitatively in rats. The observations of allodynia were mainly the changed responses of the paralyzed limb with mechanical, thermal, and cold stimulations. However, BPAI rats lost the motor and sensory functions in the paralyzed forelimbs, and previous methods were no longer applicable. Rodrigues-Filho et $\mathrm{al}^{11}$ found that the pain threshold of bilat- 
eral hind limbs decreased after BPAI in rats. Severe peripheral nerve injury leads to neuropathic pain of injured limb and subsequently induced pain hypersensitivity in intact limbs. Therefore, mechanical and thermal pain tests in the intact hind limb were applied for behavioral observation instead of the injured forelimb.

Previous studies have proposed the possible mechanisms, and central sensitization plays an important role in the initiation and progression of neuropathic pain after BPAI. Central sensitization is the enhancement in the function of neurons and circuits in nociceptive pathways, which caused by increase in membrane excitability and synaptic efficacy or reduced inhibition. It is a manifestation of the remarkable plasticity of the somatosensory nervous system in response to activity, inflammation, and neural injury. ${ }^{16}$ Central sensitization is characterized by the long-term potentiation (LTP) of neuron. After peripheral nerve injury, damaged and non-damaged A- and C-fibers begin to generate spontaneous action potentials, which is a form of ectopic input. Such input in A- and C-fibers begins to drive central sensitization in the dorsal horn of spinal cord. ${ }^{11}$ Then injured and non-injured sensory neurons in dorsal root ganglion change their membrane properties and transmitter function. ${ }^{17}$ Factors such as substance $\mathrm{P}$, brain-derived neurotrophic factor (BDNF), and the synthetic enzymes for tetrahydrobiopterin, an essential cofactor for NOS, are released and drive central sensitization. The previous study also found that A- $B$ fibers sprout into laminae I-II of the dorsal horn and make contact with nociceptive-specific neurons. ${ }^{11}$ Then nociceptive-specific neuron, which receives noxious stimuli normally, begins to receive input from non-noxious tactile stimuli and generate pain. ${ }^{18}$ The previous study also found that spinal glial cells, such as astrocyte and microglia, contribute to the induction of C-fiber LTP via NMDA receptor. ${ }^{19}$ The reduction of the synthesis, release, and activity of inhibitory transmitters also contribute to central sensitization. The death of inhibitory interneurons in the dorsal horn of spinal cord partly contributes to the loss of GABAergic and decreased glycinergic inhibitory current. ${ }^{20}$

Cortical plasticity may also take part in the process of neuropathic pain. After peripheral nerve injury, the brain representation maps in cortical and subcortical areas rearrange. Falconer ${ }^{21}$ found that rhizotomy could not improve the phantom limb pain of BPA patient, while cordotomy and dorsal root entry zone procedure could improve it. However, functional magnetic resonance image (fMRI), transcranial magnetic stimulation (TMS), and electrophysiological study still need to further investigate the relationship between cortical plasticity and neuropathic pain after BPAI.

Teixeira et al proposed that BPAI contributes to a direct injury of the spinal cord. On the same side and contralateral to the lesion, the loss of neuron in the dorsal horn was observed at various levels. ${ }^{22}$ Neuronal death may contribute to this phenomenon via the release of cytokines and chemokines, glial cells activation, and neuronal apoptosis. ${ }^{23,24}$

In our modified BPAI model, the changes in pain threshold of bilateral hind limbs was observed and assessed. These finding are consistent with previous studies. ${ }^{11,12}$

\section{Activation of astrocyte and microglia in neuropathic pain}

Glial cell, which makes up 70\% of all cell types in CNS, is an essential part of CNS. Astrocyte, oligodendroglia, and microglia are the three kinds of glial cells. ${ }^{25}$ Early researches considered that the occurrence and modulation of pain are associated with neurons; glial cells are closely involved in the process of pain. ${ }^{26}$ Since the early 1990 s, scientists found that glial cells are also involved in some information communication processes such as signal transduction, receptor expression, and transmitters expression. ${ }^{25}$ It was also reported that glial cells, especially astrocyte and microglia, play a crucial role in neuropathic pain. ${ }^{27}$ Studies demonstrated that microglia and astrocytes in the spinal cord are activated to express pro-inflammatory cytokines, chemokines, and nerve active substance, which resulted in hyperexcitability of neurons in the spinal dorsal horn. ${ }^{28}$

Microglia are activated at the early stage after BPAI and appeared to play an important role in the formation of the pain. In neuropathic pain status, microglia are rapidly evoked and the morphology, proliferative potential, gene expression, and function of microglia are changed. Microglia express several receptors for neurotransmitters, including glutamate receptors, GABA receptors, cholinergic receptors, adrenoreceptors, dopamine receptors, and purinoceptors. Other receptors include the receptors for histamine, opioids, cannabinoids, substance P, neurotrophins, chemokines, interleukins (ILs), interferons, tumor necrosis factor (TNF- $\alpha$ ), and pathogenassociated microbial patterns. Activation of the microglial receptors leads to cellular signal transduction through many essential intracellular pathways such as MAP kinases (MAPKs), nuclear factor (NF)- $\mathrm{B}$, JAK/STATs, and PI3K/ AKt. Recent studies have revealed that inhibition of some important microglial pathways is an effective way to diminish neuropathic pain states. ${ }^{29}$ Paszcuk et $\mathrm{al}^{7}$ used cannabinoid agonists to inhibit NF- $\kappa$ B pathway to relieve neuropathic pain. 
Astrocytes were activated by cytokines released from microglia and neurons. It seemed that astrocytes were more closely associated with the maintenance of neuropathic pain than microglia. In neuropathic pain status, the MAPK in astrocyte is activated by the pro-inflammatory cytokines, TNF- $\alpha$, and produces CCL2, which is critical for the development and maintenance of neuropathic pain. ${ }^{30,31} \mathrm{Lu}$ et $\mathrm{al}^{32}$ demonstrated that TNF receptor-associated factor 6 (TRAF6) upregulated in spinal cord astrocytes in the late phase after nerve injury, which maintained neuropathic pain by integrating TNF- $\alpha$ and IL- $1 \beta$ signaling and activating the JNK/CCL2 pathway in astrocytes.

In our study, the activation of glial cell was consistent with previous studies. However, in our study, the mechanism by which astrocytes were activated in the affected side, while microglia were activated in the bilateral spinal cord still need further investigation. Therefore, we can investigate the mechanism and treatment of neuropathic pain followed BPAI by using this model.

\section{Conclusion}

In the present study, we successfully established a modified model of neuropathic pain in rats by producing BPAI. Astrocyte and microglia were obviously involved in the formation and progress of neuropathic pain. Further investigations on the glial cells may provide a potentially rewarding method for treating neuropathic pain.

\section{Abbreviations}

BPAI, brachial plexus avulsion injury; GFAP, glial fibrillary acidic protein; Iba-1, ionized calcium-binding adaptor molecule 1; CCI, chronic constriction injury; PSNL, partial sciatic nerve ligation; SNL, spinal nerve ligation; SNI, spared nerve injury; CNS, central nervous system.

\section{Acknowledgments}

The authors wish to acknowledge the contribution of all the participants who took part in this study. Xu-Yun Hua performed the research and obtained the data. Mou-Xiong Zheng analyzed the data and revised the manuscript.

\section{Author contributions}

All authors contributed to data analysis, drafting and revising the article, gave final approval of the version to be published, and agree to be accountable for all aspects of the work.

\section{Disclosure}

There has been no significant financial support for this work that could have influenced its outcome. The authors report no other conflicts of interest in this work.

\section{References}

1. Qiu TM, Chen L, Mao Y, et al. Sensorimotor cortical changes assessed with resting-state fMRI following total brachial plexus root avulsion. $J$ Neurol Neurosurg Psychiatry. 2014;85(1):99-105.

2. Bruxelle J, Travers V, Thiebaut JB. Occurrence and treatment of pain after brachial plexus injury. Clin Orthop Relat Res. 1988;237(237):87-95.

3. Kretschmer T, Ihle S, Antoniadis G, et al. Patient satisfaction and disability after brachial plexus surgery. Neurosurgery. 2009;65(4 Suppl):A189-A196.

4. Baron R. Mechanisms of disease: neuropathic pain--a clinical perspective. Nat Clin Pract Neurol. 2006;2(2):95-106.

5. von Hehn CA, Baron R, Woolf CJ. Deconstructing the neuropathic pain phenotype to reveal neural mechanisms. Neuron. 2012;73(4):638-652.

6. Garrison CJ, Dougherty PM, Kajander KC, Carlton SM. Staining of glial fibrillary acidic protein (GFAP) in lumbar spinal cord increases following a sciatic nerve constriction injury. Brain Res. 1991;565(1):1-7.

7. Paszcuk AF, Dutra RC, da Silva KA, Quintão NL, Campos MM, Calixto JB. Cannabinoid agonists inhibit neuropathic pain induced by brachial plexus avulsion in mice by affecting glial cells and MAP kinases. PLoS One. 2011;6(9):e24034.

8. Hargreaves K, Dubner R, Brown F, Flores C, Joris J. A new and sensitive method for measuring thermal nociception in cutaneous hyperalgesia. Pain. 1988;32(1):77-88.

9. Seltzer Z, Dubner R, Shir Y. A novel behavioral model of neuropathic pain disorders produced in rats by partial sciatic nerve injury. Pain. 1990;43(2):205-218.

10. Kehl LJ, Trempe TM, Hargreaves KM. A new animal model for assessing mechanisms and management of muscle hyperalgesia. Pain. 2000;85(3):333-343.

11. Rodrigues-Filho R, Santos AR, Bertelli JA, Calixto JB. Avulsion injury of the rat brachial plexus triggers hyperalgesia and allodynia in the hindpaws: a new model for the study of neuropathic pain. Brain Res. 2003;982(2):186-194.

12. Gary J, Bennett Y-KX. A peripheral mononeuropathy in rat that produces disorders of pain sensation like those seen in man. Pain. 1998;33:30.

13. Decosterd I, Woolf CJ. Spared nerve injury: an animal model of persistent peripheral neuropathic pain. Pain. 2000;87(2):149-158.

14. Challa SR. Surgical animal models of neuropathic pain: Pros and Cons. Int J Neurosci. 2015;125(3):170-174.

15. Kim SH, Chung JM. Sympathectomy alleviates mechanical allodynia in an experimental animal model for neuropathy in the rat. Neurosci Lett. 1991;134(1):131-134.

16. Latremoliere A, Woolf CJ. Central sensitization: a generator of pain hypersensitivity by central neural plasticity. J Pain. 2009;10(9): 895-926.

17. Costigan M, Befort K, Karchewski L, et al. Replicate high-density rat genome oligonucleotide microarrays reveal hundreds of regulated genes in the dorsal root ganglion after peripheral nerve injury. BMC Neurosci. 2002;3:16.

18. Vrinten DH, Kalkman CJ, Adan RA, Gispen WH. Neuropathic pain: a possible role for the melanocortin system? Eur J Pharmacol. 2001;429(1-3):61-69.

19. Ma JY, Zhao ZQ. The involvement of glia in long-term plasticity in the spinal dorsal horn of the rat. Neuroreport. 2002;13(14):1781-1784.

20. Scholz J, Broom DC, Youn DH, et al. Blocking caspase activity prevents transsynaptic neuronal apoptosis and the loss of inhibition in lamina II of the dorsal horn after peripheral nerve injury. J Neurosci. 2005;25(32):7317-7323.

21. Falconer MA. Surgical treatment of intractable phantom-limb pain. $\mathrm{Br}$ Med J. 1953;1(4805):299-304.

22. Teixeira MJ, da Paz MG, Bina MT, et al. Neuropathic pain after brachial plexus avulsion-central and peripheral mechanisms. BMC Neurol. 2015;15:73

23. Ramesh G, Maclean AG, Philipp MT. Cytokines and chemokines at the crossroads of neuroinflammation, neurodegeneration, and neuropathic pain. Mediators Inflamm. 2013;2013:480739-20. 
24. Vallejo R, Tilley DM, Vogel L, Benyamin R. The role of glia and the immune system in the development and maintenance of neuropathic pain. Pain Pract. 2010;10(3):167-184.

25. Mashanov VS, Zueva OR, Garcia-Arraras JE. Organization of glial cells in the adult sea cucumber central nervous system. Glia. 2010;58(13):n/a-1593.

26. Treede RD, Jensen TS, Campbell JN, et al. Neuropathic pain: redefinition and a grading system for clinical and research purposes. Neurology. 2008;70(18):1630-1635.

27. Gosselin RD, Suter MR, Ji RR, Decosterd I. Glial cells and chronic pain. Neuroscientist. 2010;16(5):519-531.

28. Lau LT, Yu AC, Ac Y. Astrocytes produce and release interleukin-1, interleukin-6, tumor necrosis factor alpha and interferon-gamma following traumatic and metabolic injury. J Neurotrauma. 2001;18(3): $351-359$
29. Popiolek-Barczyk K, Mika J. Targeting the Microglial Signaling Pathways: New Insights in the Modulation of Neuropathic Pain. Curr Med Chem. 2016;23(26):2908-2928.

30. Gao YJ, Zhang L, Samad OA, et al. JNK-induced MCP-1 production in spinal cord astrocytes contributes to central sensitization and neuropathic pain. J Neurosci. 2009;29(13):4096-4108.

31. Zhuang ZY, Wen YR, Zhang DR, et al. A peptide c-Jun N-terminal kinase (JNK) inhibitor blocks mechanical allodynia after spinal nerve ligation: respective roles of $\mathrm{JNK}$ activation in primary sensory neurons and spinal astrocytes for neuropathic pain development and maintenance. $J$ Neurosci. 2006;26(13):3551-3560.

32. Lu Y, Jiang BC, Cao DL, et al. TRAF6 upregulation in spinal astrocytes maintains neuropathic pain by integrating TNF- $\alpha$ and IL- $1 \beta$ signaling. Pain. 2014;155(12):2618-2629.
Journal of Pain Research

\section{Publish your work in this journal}

The Journal of Pain Research is an international, peer reviewed, open access, online journal that welcomes laboratory and clinical findings in the fields of pain research and the prevention and management of pain. Original research, reviews, symposium reports, hypothesis formation and commentaries are all considered for publication.

\section{Dovepress}

The manuscript management system is completely online and includes a very quick and fair peer-review system, which is all easy to use. Visit http://www.dovepress.com/testimonials.php to read real quotes from published authors. 